\title{
Chinese Australians' Attitudes towards the Stigma of Mental Illness: Analysis Using Structural Equation Modelling with AMOS
}

\author{
Tan Kan Ku${ }^{1}$, Michael $\mathrm{Ha}^{2 *}$ \\ ${ }^{1}$ Institute of Health and Management, IHM, Melbourne, Australia \\ ${ }^{2}$ College of Global Talents, Beijing Institute of Technology, Zhuhai, China \\ Email: dr.tan@healthcareers.edu.au, tankanku@gmail.com, ${ }^{*}$ Michael.Ha@cgt.bitzh.edu.cn
}

How to cite this paper: Ku, T.K. and Ha, M. (2021) Chinese Australians' Attitudes towards the Stigma of Mental Illness: Analysis Using Structural Equation Modelling with AMOS. Journal of Biosciences and Medicines, 9, 182-193.

https://doi.org/10.4236/jbm.2021.96017

Received: May 26, 2021

Accepted: June 27, 2021

Published: June 30, 2021

\begin{abstract}
Aim: The quantitative study aims to elucidate the relationship between contact factor with someone who has a mental illness, cultural values, and differential approaches towards mental illness (depression versus schizophrenia) among 138 Chinese immigrants in Australia. The study results aim to provide community mental health literacy program to immigrants in Australia that seek to lessen their attitudes of stigma towards mental illness. Method: A cross-sectional survey (General Population Questionnaire) comprising of 118 items) was administered to 138 Chinese immigrants ( 96 female and 42 male) to examine their Cultural Affiliation (Chinese versus Australian Identification). Factor analyses were conducted to examine the discriminant validity of the subscales. Findings: Chinese Australians endorsed more Chinese Culture Identification than Australian Culture Identification. These immigrants endorsed more highly collectivist values than individualistic values. Stigma factors (social distancing and negative stereotyping attitudes) correlated negatively with readiness to give support to the depression vignette than the schizophrenia vignette. Pearson's correlation matrix and Hierarchical Regression Analyses when cultural values and vignette approaches were considered as influencing factors-details of the subsequent findings will be reported in another paper. Conclusion: Several Structured Equation Models suggested that attitudinal approaches towards someone with a mental illness and stigma are associated with Contact level and Cultural Affiliation. Contact is better placed as a mediating factor.
\end{abstract}

\section{Keywords}

Mental Illness, Culture, Stigma, Structure Equation Modelling 


\section{Introduction}

\section{Background}

This paper provides the analysis of 138 Chinese Australians' attitudes towards mental illness and whether acculturation has an effect on stigma. As part of the design of a PhD study of Chinese immigrants' cultural values, their contact level with people who have a mental illness and whether Saving face, and Social Comparison affect their attitudes between a case of depression and schizophrenia in vignette format. The sample compromised of Immigrants with ethnic Chinese background arriving in Australia between 1959-2009. In this paper, we report the final analysis (five Structured Equation Models by AMOS). Contact level via Conversation (CVC), Cultural factors (Collectivist Family Conformity, CFC, Individualist Self Assertion, ISA), Stigma factors (Social Distancing Attitudes, SDA, Negative Stereotype Attitudes, NSA) and how they translated into vignette approaches will be reported in another paper based on Pearson's Correction Matrix and Hierarchical Multiple Regression Analysis.

\section{Research questions:}

The following research questions were explored:

Is contact level with mental illness related with stigma of mental illness in three groups of Chinese Australians?

Is cultural affiliation (CAS) related to stigma of mental illness in three groups of Chinese Australians?

\section{Literature review}

It was well documented in the international literature/thesis that Chinese immigrants under-utilised mental health services in Australia [1] [2] [3] [4]. This was partly due to Chinese indigenous way of conceptualising mental illness and attributing it to "fate"; a preference to somatising as "normal physical symptom" [5]. Reluctance to seek help could be related to "saving face" [6].

A study conducted by Papadopoulos et al. [7] in the United Kingdom to examine whether cultural values (based on the Individualism-Collectivism Construct) had an impact on English, Chinese and Greek's attitudes towards mental Illness. The results suggested that more positive cultural attitude towards mental illness the more likely explanation was associated with the individualism paradigm, and that the Chinese group's greater stigmatising attitudes was related to collectivism.

A local cross-cultural study completed by Mellor et al. [8] suggested that Chinese immigrants and Taiwanese held more stigmatising attitudes towards mental illness than Australian-born Chinese and Anglo-Australians. The authors commented that this might be due to differential cultural values related to individualism and collectivism in Western culture and Chinese culture.

The majority of studies related to attitude used quantitative methodology to measure stigma. Diversified contacts with people with a mental illness [9] [10] lessen stigma measured using the Social Distancing Scale and improved attitude towards the perception of mental illness [11]. 


\section{Method and Design}

The General Population Questionnaire (available on request from the first author) was administered in English Language. A quantitative approach was chosen in the design of the study because using number (statistical data) is a unique way of conceptualising/contextualising attitude study for the perception of mental illness stigma. Prior to key analyses, scales were examined for their psychometric properties and further refinement.

\subsection{Setting}

The study was conducted with ethnic Chinese people living in Metropolitan Melbourne. Melbourne is the second largest city in Australia with a diverse multi-cultural population of six and a half million [12]. Australia's Asian population is soaring, and ethnic Chinese people are among the fastest growing immigrants behind the United Kingdom and New Zealand [12].

\subsection{Ethical Consideration}

Ethics approval (Project No. 26/09) was granted from the RMIT University Human Ethics Committee in 2009-2013. Prior to participating in the study, potential participants were required to read the Project Information Statement/ Plain Language Statement which indicated that by completing and returning the questionnaire in the Reply-paid envelope, their consents were implied.

\subsection{Response Rate}

Two hundred and fifty-three questionnaires were distributed either in person or via mail (each envelope containing six questionnaires) after initial contact with the prospective participants by the first author. One hundred and forty-four were returned (response rate of 56.9\%) and six of these questionnaires were not completed. So, 138 participants out of the 144 relevant respondents returned the questionnaire giving a response rate of $54 \%(138 / 247 \times 100)$.

\subsection{Data Collection}

Adults who self-identified as ethnic Chinese and resident in Melbourne, Australia were approached to participate in the study. To avert bias in snowballing, an initial large pool of Chinese immigrants $(n=20)$ from different professional and social backgrounds was identified to be asked to participate in the study and to provide access to other Chinese people. Age range was from 18 - 65.

\subsection{Sample Characteristics}

From the sample of 138 , there were only four subjects (2.9\%) were born locally in Australia of immigrant parents. The other 134 subjects (97.1\%) were all immigrants in Australia. Participants were mainly professionals working in the health sector (including nurses, doctors and social workers accounting for 40.6\%), commercial, legal, information technology and engineering (34.1\%), teaching 
and learning sectors including teachers, tertiary students, researchers and translators (21.6\%). The sample was biased towards female (96/69.6\%) compared with male $(42 / 30.4 \%)$. The mean age was 39.3 . Due to the diverse age range of participants $(\mathrm{n}=138)$, age was specified into three categories from the youngest to the oldest age group: Age Group 1, AGE1 (18 - 34), Age Group 2, AGE2 (35 - 49) and Age Group 3, AGE3 (50+). Exact p-values for the Chi-Square test of association were examined using Monte Carlo methods when the assumption of less than $20 \%$ of cells having expected counts below five was violated. Otherwise, Chi-square asymptotic p-values were reported. Table 1 indicates the decade of arrival of ethnic Chinese immigrants in Australia.

Since the number of arrivals was not even, the decade of arrival was re-specified into three categories: DECOA1 (1950-1979), DECOA2 (1980-1999) and DECOA3 (2000-2009) attempting to have even number of participants for group comparisons at a later stage. Hence, there were 25 arrivals during DECOA 1, 53 in DECOA2 and 56 in DECOA3.

\subsection{Data Analysis}

SPSS (Statistical Analysis for Social Sciences) Version 20 was used for statistical analysis. Descriptive statistics was used for sample demographics (Table 2). Analysis of Variance (ANOVA) was conducted on group data including groups comparisons, and pair sample t-Tests, scale construction/refinement methods including factor analysis, item-homogeneity analyses, and correlation method including Pearson corrections. Confidence interval was set at $95 \%$. With categorical data, for sample description, additional contingency table analyses were conducted using the Chi-square statistics. For scale development, a principal component analysis (PCA) was conducted on the items of the ADSS [13] and the CVS [14]. Table 3 shows the Pattern Matrix for the CAS Scale.

The first factor was labelled Chinese Culture Identification (CCI) as items indicated participants' attachment to parental culture. The second factor was labelled Australian Culture Identification (ACI) suggesting how individual's adoption of Australian way of living. Treating each of the factors as scales, reliability analysis indicated relatively high internal consistency among scale items. Alpha coefficients are shown in Table 3.

Table 1. Number of Chinese and Period of Arrival in Australia.

\begin{tabular}{cc}
\hline Period of Arrival & Number of Immigrant Chinese Frequency (\%) \\
\hline $1950-1959$ & $4(2.9)$ \\
$1960-1969$ & $3(2.2)$ \\
$1970-1979$ & $18(13)$ \\
$1980-1989$ & $38(27.5)$ \\
$1990-1999$ & $15(10.9)$ \\
$2000-2009$ & $56(40.6)$ \\
\hline
\end{tabular}


Table 2. Characteristics of the Sample $(\mathrm{N}=138)^{\#}$.

\begin{tabular}{|c|c|c|c|c|}
\hline & $\begin{array}{c}\text { DECOA } 1 \\
(1950-1979) \\
n=25\end{array}$ & $\begin{array}{c}\text { DECOA } 2 \\
(1980-1999) \\
n=53\end{array}$ & $\begin{array}{c}\text { DECOA } 3 \\
(2000-2009) \\
\mathrm{n}=56\end{array}$ & $\chi^{2} / F$ value \\
\hline Mean Age (SD) & $30.6(10.4)$ & $30.6(10.4)$ & $30.6(10.4)$ & $F(2,131)=41.2^{* * *}$ \\
\hline Sex (male/female) & $18 / 38$ & $18 / 38$ & $18 / 38$ & $1.5 \mathrm{~ns}$ \\
\hline Education Level (\%) & & & & $22.7^{\star \star 1}$ \\
\hline Primary & 0 & 0 & 0 & \\
\hline Secondary & 0 & 0 & 0 & \\
\hline Completed secondary & 25.0 & 25.0 & 25.0 & \\
\hline Completed trade & 5.4 & 5.4 & 5.4 & \\
\hline Undergraduate & 35.7 & 35.7 & 35.7 & \\
\hline Postgraduate & 33.9 & 33.9 & 33.9 & \\
\hline Professional role (\%) & & & & $32.9^{* * * 1}$ \\
\hline Health worker & 20.0 & 20.0 & 20.0 & \\
\hline Business & 18.2 & 18.2 & 18.2 & \\
\hline Education & 7.3 & 7.3 & 7.3 & \\
\hline Other professional & 21.8 & 21.8 & 21.8 & \\
\hline Other low income & 32.7 & 32.7 & 32.7 & \\
\hline Know someone with mental illness (\%) & & & & $10.1 \mathrm{~ns}^{1}$ \\
\hline Friend & 58.9 & 58.9 & 58.9 & \\
\hline Spouse & 0 & 0 & 0 & \\
\hline Sibling & 0 & 0 & 0 & \\
\hline Parents & 0 & 0 & 0 & \\
\hline Colleague & 7.1 & 7.1 & 7.1 & \\
\hline Relative & 32.1 & 32.1 & 32.1 & \\
\hline
\end{tabular}

DECOA: Decade of Arrival; ${ }^{*}$ Four subjects were born locally; ${ }^{* *} \mathrm{p}<0.01,{ }^{* * *} \mathrm{p}<0.001$; ns, not significant; ${ }^{1}$ Exact $\mathrm{p}$-value.

Table 3. Pattern Matrix for the CAS Scale (Cultural Affiliation Scale)

\begin{tabular}{cccc}
\hline Item & F1 & F2 & Communality \\
\hline QG6 extent of maintaining parents' culture & 0.90 & 0.01 & 0.80 \\
QG3 feel to be of parents' culture & 0.89 & -0.08 & 0.80 \\
QG2 how important to be of parents' culture & 0.87 & 0.07 & 0.79 \\
QG1 how Australian do you feel & -0.15 & 0.86 & 0.77 \\
QG4 how important is being Australian & 0.12 & 0.86 & 0.75 \\
QG5 extent of adopting Australian way & 0.01 & 0.86 & 0.74 \\
Percent variance & 40.5 & 36.9 & Total variance $=77.4$ \\
Cronbach's alpha & 0.87 & 0.82 & \\
\hline
\end{tabular}

F1: CCI; F2: ACI.

\subsection{Group Contrasts on Cultural Affiliation Scores}

Two-way analysis of variance was conducted to examine differences between age group and decade of arrival. The first analysis examined Chinese Culture Identi- 
fication (CCI) scores. The main effect was statistically significant for age group $(\mathrm{F}(2,125)=3.87, \mathrm{p}<0.05)$ and decade of arrival $(\mathrm{F}(2,125)=4.14, \mathrm{p}<0.05)$, but not the interaction term $(\mathrm{F}(4,125)=0.58, \mathrm{p}>0.05)$ indicating that Chinese Culture Identification did not vary across age group and decade of arrival. Post Hoc testing using Tukey HSD procedure $(\alpha=0.05)$ showed no statistical significance. Examination of the mean showed that Chinese people who arrived in Australia earlier (1959-1979) endorsed higher Chinese culture identification $($ mean $=9.12$, s.d. $=1.90)$ than the latter group $($ mean $=8.72$, s.d. $=2.14)$ for those arriving during Decade 2 (1980-1999) and for the most recent migrants $($ mean $=8.23$, s.d. $=1.97)$ who arrived during Decade 3 (the period of 20002009) respectively. Examination of the age differences indicated that the younger age group endorsed higher level of Chinese culture identification (mean $=8.77$, s.d. $=2.04$ ) for the $18-34$ group than the $35-49$ age group (mean $=8.67$, s.d. $=$ $1.98)$ and the oldest age group $(50+)($ mean $=8.30$, s.d. $=2.09)$. The overall mean score was 2.85 (s.d. $=0.68$ ) indicating that on average, affiliation with Chinese culture was between "a little" and "much" but favouring the latter response category. In addition, $57.3 \%$ of the sample had an item average of greater than 3 , indicating that they either favour the "much" or "very much" responses.

The second analysis focused on examining Australian Culture Identification (ACI) scores. The main effect of age group was not significant $(\mathrm{F}(2,125)=2.06$, $\mathrm{p}>0.05)$. However the main effect of decade of arrival was statistically significant $(\mathrm{F}(2,125)=3.65, \mathrm{P}<0.05)$. Post Hoc testing using Tukey HSD procedure $(\alpha=0.05)$ indicated significance for two of the three pairwise comparisons. Specifically, DECOA 1 (1959-1979) was significantly different to DECOA 2 ( $\mathrm{p}<$ $0.001)$ and DECOA $3(\mathrm{p}<0.001)$. Inspection of the means indicated that the early migrants (arriving 1950-1979) endorsed higher level of Australian culture identification (mean $=8.76$, s.d. $=1.94$ ) than the more recent arrival for $1980-1999($ mean $=7.92$, s.d. $=1.85)$ and for those arriving 2000-2009 $($ mean $=$ 6.38 , s.d. $=1.76)$. The interaction term was not statistically significant $(F(4,125)$ $=0.58, \mathrm{p}>0.05)$ indicating that Australian culture identification did not vary across age group. The overall mean score was 2.50 (s.d. =0.69) implying that the average rating was at the centre of the scale between "a little" and "much". In addition, $29.7 \%$ of the sample favouring an item average of between 3 and 4 indicating that they either favoured "much" or "very much" responses. Overall results suggest that the three groups of Chinese immigrants endorsed a stronger affiliation with their origin Chinese culture. This correlates positively with what had been documented in the literature and confirm that majority of Chinese immigrants in the Western world maintain their ethnic culture.

\section{Discussion}

\section{Relationship between Cultural Values, Contact and Attitudinal Approaches among Chinese Australians}

Previous analyses (Pearson's and Hierarchical Regression) dealt with 3 groups 
membership indicating a relationship between contact, collectivist values (CFC $\&$ CFI) and individualist value (ISA) and SUPP_A are associated with stigma (SDA \& NSA). In addition, SOAD_G is also related to NSA. Structural Equation Modelling (SEM) [15] [16] was then applied to strengthen the research findings for investigating the constructs that underlie such sets of measures.

Researchers all understand one of the most important stages in research projects is the construction of a model. Model-building is both science and art which require a thorough understanding of the topic and the use of statistics. A researcher must

1) Identify all potential factors that exist in a model;

2) Assess the importance of these factors;

3) Measure their influence in the model;

4) Manage these factors by correctly combining them since not all factors are used in the final model;

5) Re-examine the validity of the combined factors; and

6) Interpret statistical results by referring to the chosen and combined factors.

The construction of a model requires time, patience and experience. It is also a result of testing, trial and error. At a starting point, correlations among these measures were mapped. That is, cultural values, contact factor and vignette approaches (CFC, CVC, ISA, SUPP_A, SOAD_G) were considered as independent variables (referred to as exogenous variables) (as per AMOS Output) and stigma (SDA and NSA) as dependent variables (referred to as endogenous variables) (as per AMOS Output) [16]. The next step is to establish a null hypothesis $=$ There is no difference between $\hat{\Sigma}$ and $S$ to calculate estimates for the model parameters. Figures 1-5 indicate several path analyses conducted with AMOS-20 [15] and conclude with Figure 5 as the best model. Some of the results of repeated testing are provided in Figure 1.

At $\mathrm{p}($ or $\alpha)=0.05$, and $v=2$, the critical value is 5.9914. $\chi_{0.05,2}^{2}=5.9914$. Chi-square test statistics is 40.268 which is greater than the critical value, 3.841 , we reject the null hypothesis. There is sufficient evidence to reject the Null Hypothesis as 40.27 is on the RHS (right-hand-side) of the Critical Value, 5.9914. We conclude that the difference between $\hat{\Sigma}$ and $S$ is due to chance alone $(0.00 \%)$ and conclude that the hypothesis that $\hat{\Sigma}$ and $S$ are not significantly different is untenable. RMSEA $=0.374$ indicates a poor fit.

At $\mathrm{p}($ or $\alpha)=0.05$, and $v=1$, the critical value is 3.841. $\chi_{0.05,1}^{2}=3.841$. Chisquare test statistics is 39.250 which is greater than the critical value, 3.841, we reject the null hypothesis. There is sufficient evidence to reject the Null Hypothesis. We can see that 39.250 is on the RHS (right-hand-side) of the Critical Value, 3.841. We conclude that the difference between $\hat{\Sigma}$ and $S$ is due to chance alone $(0.00 \%)$ and conclude that the hypothesis that $\hat{\Sigma}$ and $S$ are not significantly different is untenable. RMSEA $=.528$ indicates a poor fit. 


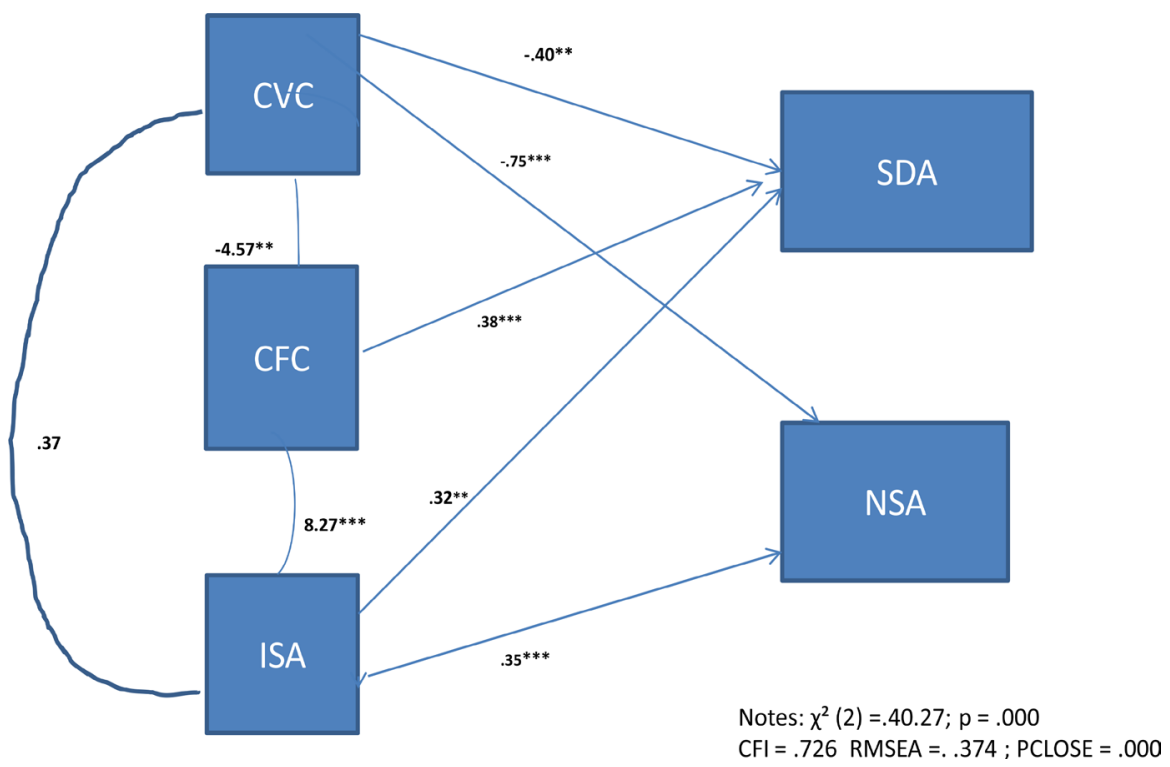

Figure 1. Path Analysis Model 1-Relationship between contact, cultural values and stigma.

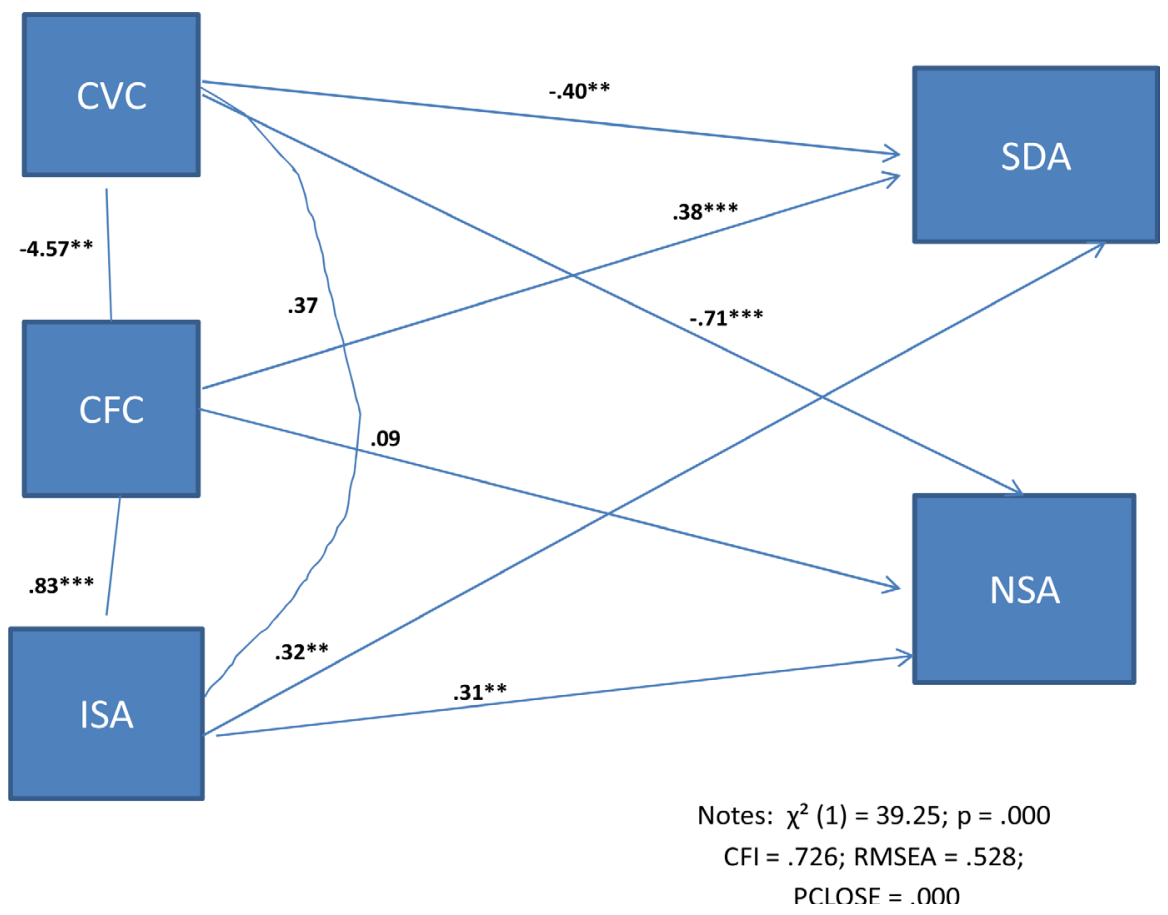

Figure 2. Path Analysis Respecified Model 2-Relationship between contact, cultural values and stigma.

At $\mathrm{p}($ or $\alpha)=0.05$, and $v=1$, the critical value is 3.841. $\chi_{0.05,1}^{2}=3.841$. Chisquare test statistics is 28.194 which is greater than the critical value, 3.841 , we reject the null hypothesis. There is sufficient evidence to reject the Null Hypothesis. We can see that 28.194 is on the RHS (right-hand-side) of the Critical Value, 3.841. We conclude that the difference between $\hat{\Sigma}$ and $S$ is due to chance alone $(0.00 \%)$ and conclude that the hypothesis that $\hat{\Sigma}$ and $S$ are not significantly different is untenable. RMSEA $=0.446$ indicates a poor fit. 


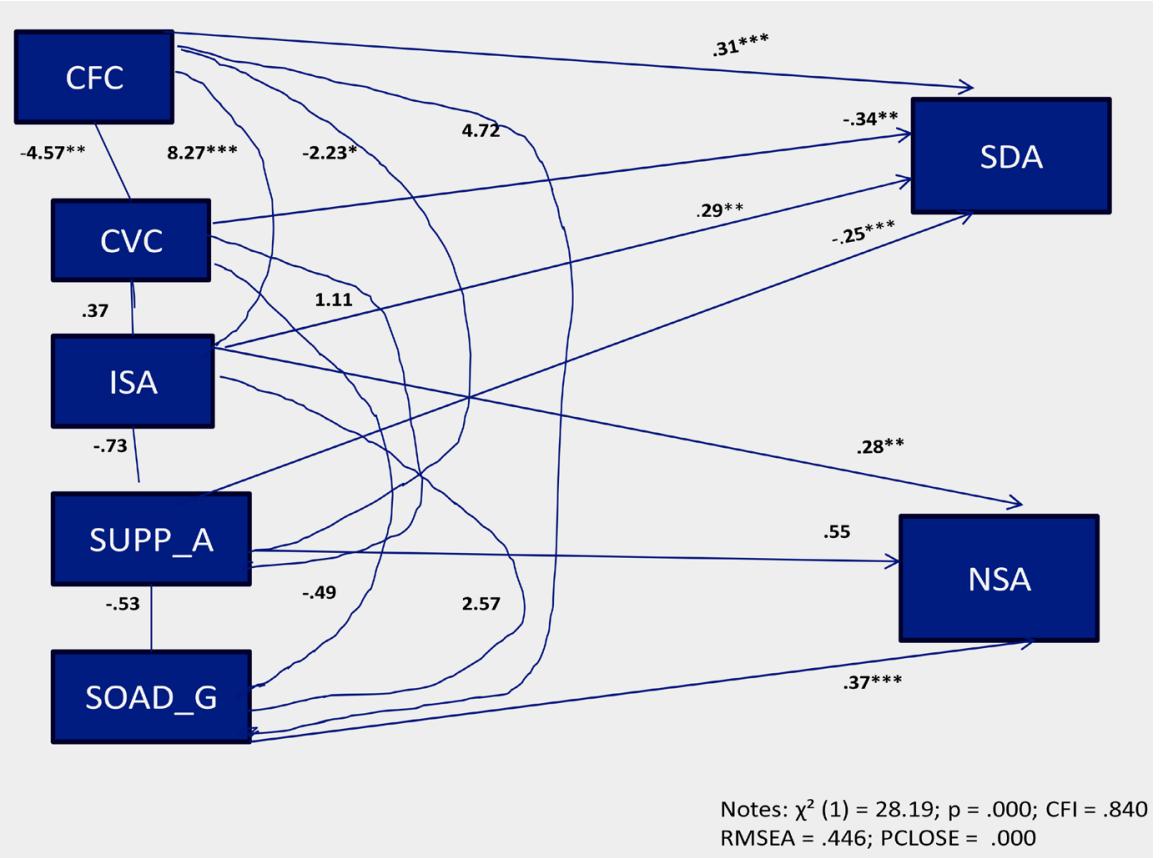

Figure 3. Path Analysis Respecified Model 3-Relationship between contact, cultural values, vignette approaches and stigma.

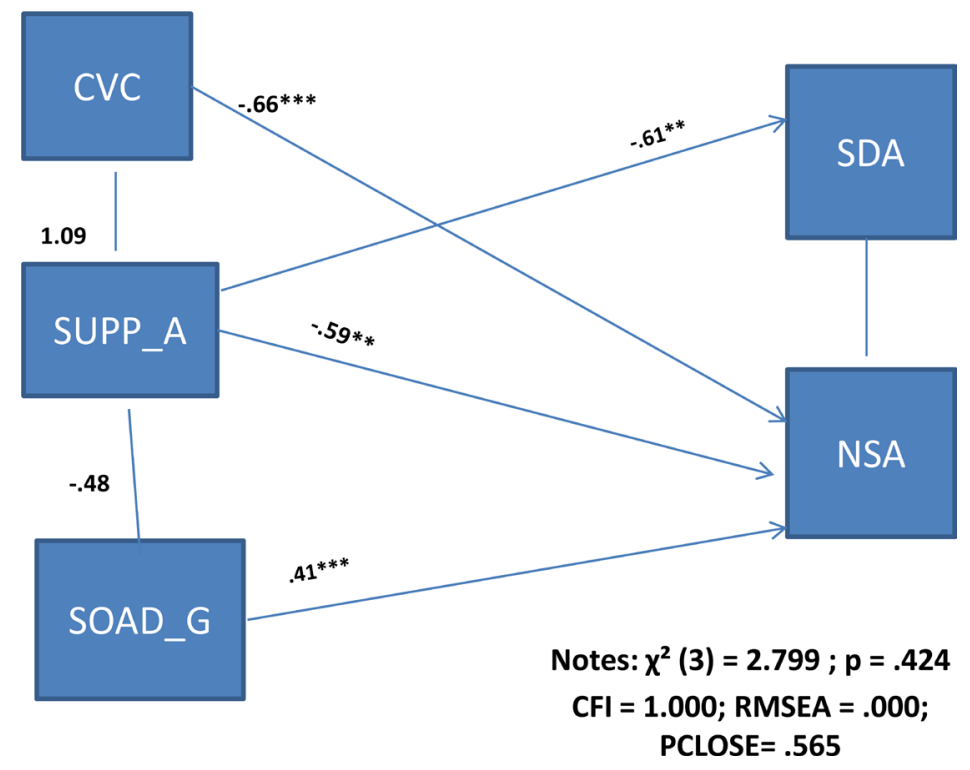

Figure 4. Path Analysis Respecified Model 4-Relationship between contact, vignette approaches and stigma with the removal of cultural values.

At $\mathrm{p}($ or $\alpha)=0.05$, and $v=3$, the critical value is 7.8147. $\chi_{0.05,3}^{2}=7.8147$. Chi-square test statistics is 2.799 which is less than the critical value, 7.8147, we do not reject the null hypothesis. We do not have sufficient evidence to reject the Null Hypothesis. We can see that 2.799 is on the LHS (left-hand-side) of the Critical Value, 7.8147. We conclude that the difference between $\hat{\Sigma}$ and $S$ is due to chance alone $(42.36 \%)$ and conclude that the hypothesis that $\hat{\Sigma}$ and S are not significantly different is tenable. RMSEA $=0.000$ indicates the best fit. 


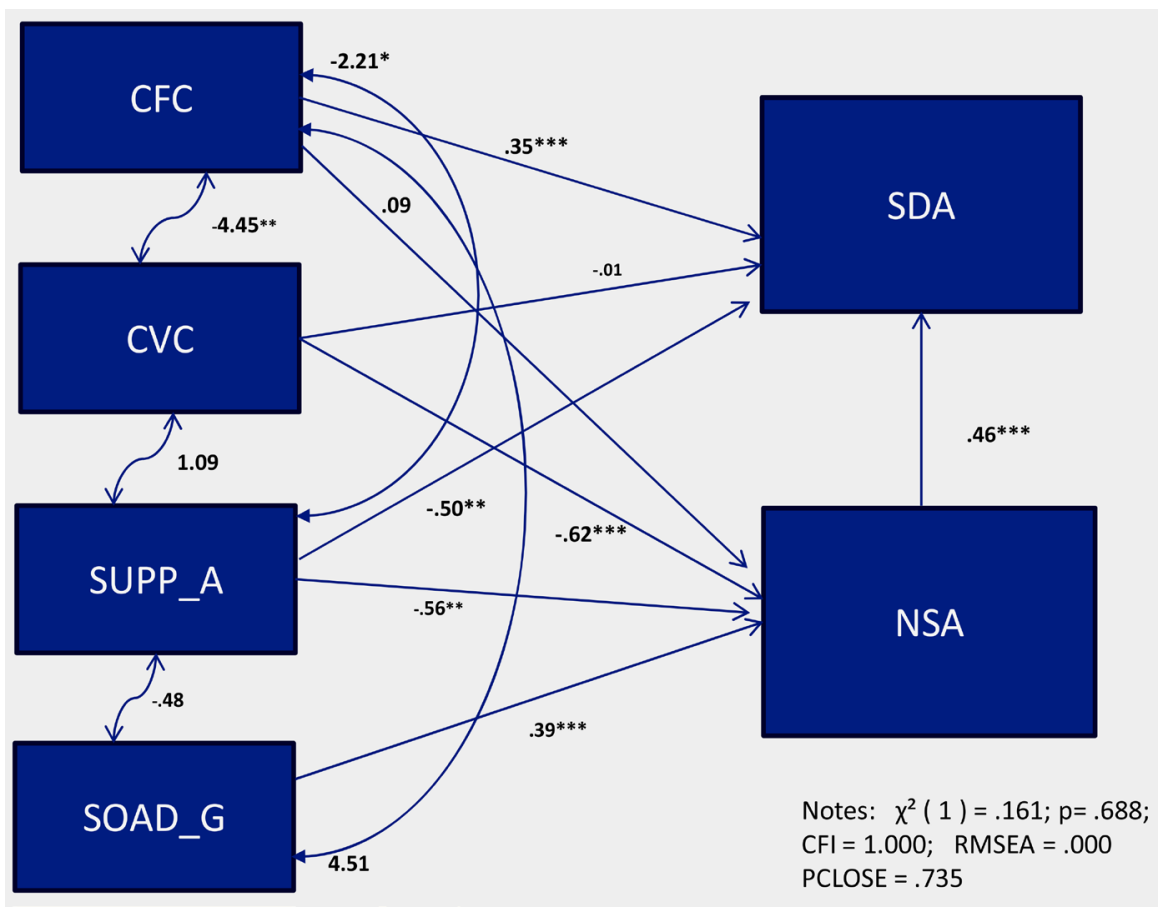

Figure 5. Path Analysis Respecified Model 5-Relationship between contact, collectivist value, vignette approaches and stigma.

At $\mathrm{p}($ or $\alpha)=0.05$, and $v=1$, the critical value is 3.841. $\chi_{0.05,1}^{2}=3.841$. Chisquare test statistics is 0.161 which is less than the critical value, 3.841 , we do not reject the null hypothesis. We do not have sufficient evidence to reject the Null Hypothesis as 0.161 is on the LHS (Left-Hand-Side) of the Critical Value. We conclude that the difference between $\hat{\Sigma}$ and $\mathrm{S}$ is due to chance alone (68.82\%) and conclude that the hypothesis that $\hat{\Sigma}$ and $\mathrm{S}$ are not significantly different is tenable. RMSEA $=0.000$ indicates the best fit.

Regardless of the details of the various alternative models (poor versus good fits), the main general results suggest that Chinese values (particularly Collectivist Family Conformity, CFC and Individualistic Self Assertion, ISA) are related to stigma, and especially Social Distancing Attitudes. Contact level was expected to influence social distancing and negative stereotyping attitudes, given the casual relationships in the literature in decreasing stigmatising attitudes, the results in the current study indicate that it is better placed as a mediator between negative stereotyping attitudes and attitudinal approaches towards someone with a mental illness. The final model suggests a negative correlation between SUPP_A and Social Distancing Attitudes (SDA) and Negative Stereotyping Attitudes (NSA, also a positive correlation between SOAD_G (Potential for Social Avoidance) and NSA. Conclusively, the relationship between attitudinal approaches towards someone with a mental illness and stigma is associated with Contact level and Cultural Values.

\section{Recommendation}

Given the paucity of Australian study on public or professional attitudes towards 
mental illness in the Chinese community, future research should aim at determining the actual factor(s) influencing Chinese people's preference in adopting either their origin or host culture. Cultural affiliation may have inadvertently affect their attitudes towards mental illness, among other factors.

Doctors and nurses can be encouraged to specialise in mental health when they begin their medical/nursing training. Problem-based Learning (PBL) can be incorporated with Culture Respect Encompassing Simulation Training (CREST) as part of the teaching/learning activities [17].

\section{Conclusion}

The results support the notion that "cultural affiliation" has an impact on Chinese immigrants' attitudes towards mental illness. Findings also allow a "Yes" answer to the two research questions. Contact level and cultural affiliation are related to stigma of mental illness in the three groups of Chinese Australians. Contact factor has a mediating effect in the final analysis with AMOS. Despite the small sample size, the study had made significant contribution in mental health research in the Australian setting, as it provided new data in Melbourne, Australia.

\section{Acknowledgements}

The authors would like to thank the Institute of Health and Management (IHM) and the College of Global Talents (CGT), Beijing Institute of Technology, Zhuhai for supporting and sponsoring the publication of this paper.

\section{Conflicts of Interest}

The authors declare no conflicts of interest regarding the publication of this paper.

\section{References}

[1] Ku, T.K. (2017) Culture and Stigma towards Mental Illness: An Analysis of Chinese Immigrants' Attitudes in Australia. PhD Thesis. School of Health Science-Public Health Application, Turks and Caicos Island, West Indies, British Isles.

[2] Klimidis, S., Hsiao, F.H. and Minas, H. (2007) Chinese-Australians' Knowledge of Depression and Schizophrenia in the Context of Their Under-Utilisation of Mental Health Care: An Analysis of Labelling. The International Journal of Social Psychiatry, 53, 464-479. https://doi.org/10.1177/0020764007078357

[3] Hsiao, F.-H. (2002) Chinese-Australian Families' Help-Seeking Behaviour for Mental Illness. PhD Thesis. The University of Melbourne.

[4] Minas, I.-H., Lambert, T.I.R., Kostov, S. and Boronga, G. (1996) Mental Health Services for NESB Immigrants. Transforming Policy into Practice. Bureau of Immigration, Multicultural and Population Research. Australian Government Publishing Service, Canberra.

[5] Lin, T. (1983) Psychiatry and Chinese Culture. The Western Journal of Medicine, 139, 862-867. 
[6] Lau, Y. and Wong, D.F.K. (2007) Are Concern for Face and Willingness to Seek Help Correlated to Early Postnatal Depressive Symptoms among Hong Kong Chinese Women? A Cross-Sectional Questionnaire Survey. International Journal of Nursing Studies. https://www.e;sevier.com/locate/ijnurstu https://doi.org/10.1016/j.ijnurstu.2006.08.002

[7] Papadopoulos, C., Foster, J. and Caldwell, K. (2012) "Individualism-Collectivism" as an Explanatory Device for Mental Illness Stigma. Community Mental Health Journal, 49, 270-280. https://doi.org/10.1007/s10597-012-9534-x

[8] Mellor, D., Carne, L., Shen, Y.-C., McCabe, M. and Wang, L. (2013) Stigma towards Mental Illness: A Cross-Cultural Comparison of Taiwanese, Chinese Immigrants to Australia and Anglo-Australians. Journal of Cross-Cultural Psychology, 44, 352-364. https://doi.org/10.1177/0022022112451052

[9] Corrigan, P.W., River, L.P., Lundin, R.K., Penn, D.L., Uphoff-Wasowski, K., Campion, J., Mathisen, J., Gagnon, C., Bergman, M., Goldstein, H. and Kubiak, M.A. (2001) Three Strategies for Changing Attributions about Severe Mental Illness. Schizophrenia Bulletin, 27, 187-195.

https://doi.org/10.1093/oxfordjournals.schbul.a006865

[10] Ku, T.K., Ha, M. and Siriwan, U. (2015) Professional Attitudes towards Mental Illness: Testing the Contact Hypothesis among Nurses of Ethnic Anglo and Chinese Australian Backgrounds. Open Journal of Social Sciences, 3, 130-138. https://doi.org/10.4236/jss.2015.37022

[11] Ku, T.K. and Ha, M. (2015) Stigma of Mental Illness: Social Distancing Attitudes among Registered Nurses in Australia. Journal of Biosciences and Medicine, 3, 40-47. https://doi.org/10.4236/jbm.2015.312007

[12] Australian Bureau of Statistics (ABS) (2016) 34170DO001_Understanding Migrant Outcome-Insights from the Australian Census and Migrants Integrated Dataset, Victoria, 2016. Released at 11:30 am (Canberra Time) 18 July 2018.

[13] Ku, T.K. and Ha, M. (2015) Development of the Attitudes towards Depression and Schizophrenia Scale (ADSS). Studies in Social Sciences and Humanities, 2, 12-20.

[14] Ku, T.K. and Ha, M. (2017) Individualistic and Collectivistic Values, Age and Length of Residency among Chinese Australia Immigrants. Psychology and Behavioural Sciences, 6, 90-95.

http://article.sciencepublishinggroup.com/pdf/10.11648.j.pbs.20170605.13.pdf https://doi.org/10.11648/j.pbs.20170605.13

[15] Arbuckle, J.L. (2003) AMOS-5 (Software). Chicago. Smallwaters Core.

[16] Loxton, N. (2013) Course Notes for Applied Structured Equation Modelling Using AMOS. ACSPRI 2013 Summer Program in Social Research Methods and Research Technology. Australian Consortium for Social and Political Research Incorporated. University of Melbourne.

[17] Ku, T.K. and Ha, M. (2016) The Application of Problem Based Learning in Undergraduate Nursing Education: A Strategy for Curriculum Reform. Journal of Biosciences and Medicines, 4, 52-59. https://doi.org/10.4236/jbm.2016.46008 